\title{
A METHOD FOR EVALUATION OF THE CHAIN DRIVE EFFICIENCY
}

\author{
Aleksey Egorov* \\ Volga State University of Technology, Yoshkar-Ola, Russia \\ Konstantin Kozlov \\ Volga State University of Technology, Yoshkar-Ola, Russia \\ Vladimir Belogusev \\ Volga State University of Technology, Yoshkar-Ola, Russia
}

This paper is devoted to the development of a method and instruments for evaluation of the efficiency of a chain drive in all possible modes of operation. The proposed method allows determining the torque and the efficiency of chain drives and evaluating their efficiency with a high measurement rate as it uses the acceleration time as the efficiency criterion. A new method requires a minimal amount of additional equipment and, therefore, is more cost-effective than existing methods. Based on the results obtained at experiments we can conclude the applicability of the developed method for evaluation of the chain drive efficiency, and it can be fully used for the study and technical state control of chain drives.

Key words: Chain drive, Efficiency, Moment of inertia, Performance, Chain transmission

\section{INTRODUCTION}

Increase in the efficiency of chain drives is an important task to improve the quality of their production, since friction conditions in chain drives effect significantly the rate of degradation processes, and consequently, have a significant impact on their lifetime. The majority of publications devoted to the study of chain transmissions [01, 02, 03, 06, 08] pay little attention to the matter of measuring their energy performance. Known methods and instruments do not allow studying more accurately the mechanical losses in chain transmissions at most of speed and load operation modes, and their application is hindered by the complexity and costliness of measuring equipment [04, 05]. It is known, that chain transmissions have comparatively high values of their efficiency (92 ... $97 \%$ ) (7), and the existing methods for chain drive efficiency evaluation do not meet necessary requirements of measurement accuracy. The existing methods, in order to determine the induction motor torque, are based on either both assumption and averaging (brake methods) or on the set of indirect parameters (e.g., input and output electrical parameters). The most commonly used method of strain measurement requires calibration of strain gages, which leads to the additional errors during the measuring process.
The present paper describes a new method and methodology for evaluation of the energy efficiency of chain drives in a wide range of speed and load operation modes. The developed method is free of the above limitations and determines the efficiency by measuring the angular acceleration of the rotating masses during the speeding up of a chain drive.

\section{MATERIALS AND METHODS A Method to Determine the Efficiency of a Chain Drive}

The implementation of the method is explained on the basis of a scheme of a chain drive with an induction motor (electric chain drive) (Fig. 1). Figure 1 shows the elements, which are included in an electric chain drive. This method is based on determining the moment of inertia of rotating masses comprising the rotating units of these elements.

In order to determine the efficiency of an electric chain drive, it is necessary to find what part of the total energy consumed to accelerate a mechanical system of rotating parts is taken to overcome the friction losses in a chain mesh. That is, it is necessary to determine the moment of inertia of a chain drive with respect to the rotation axis of the drive shaft with and without losses in the mesh of a chain 7 . 

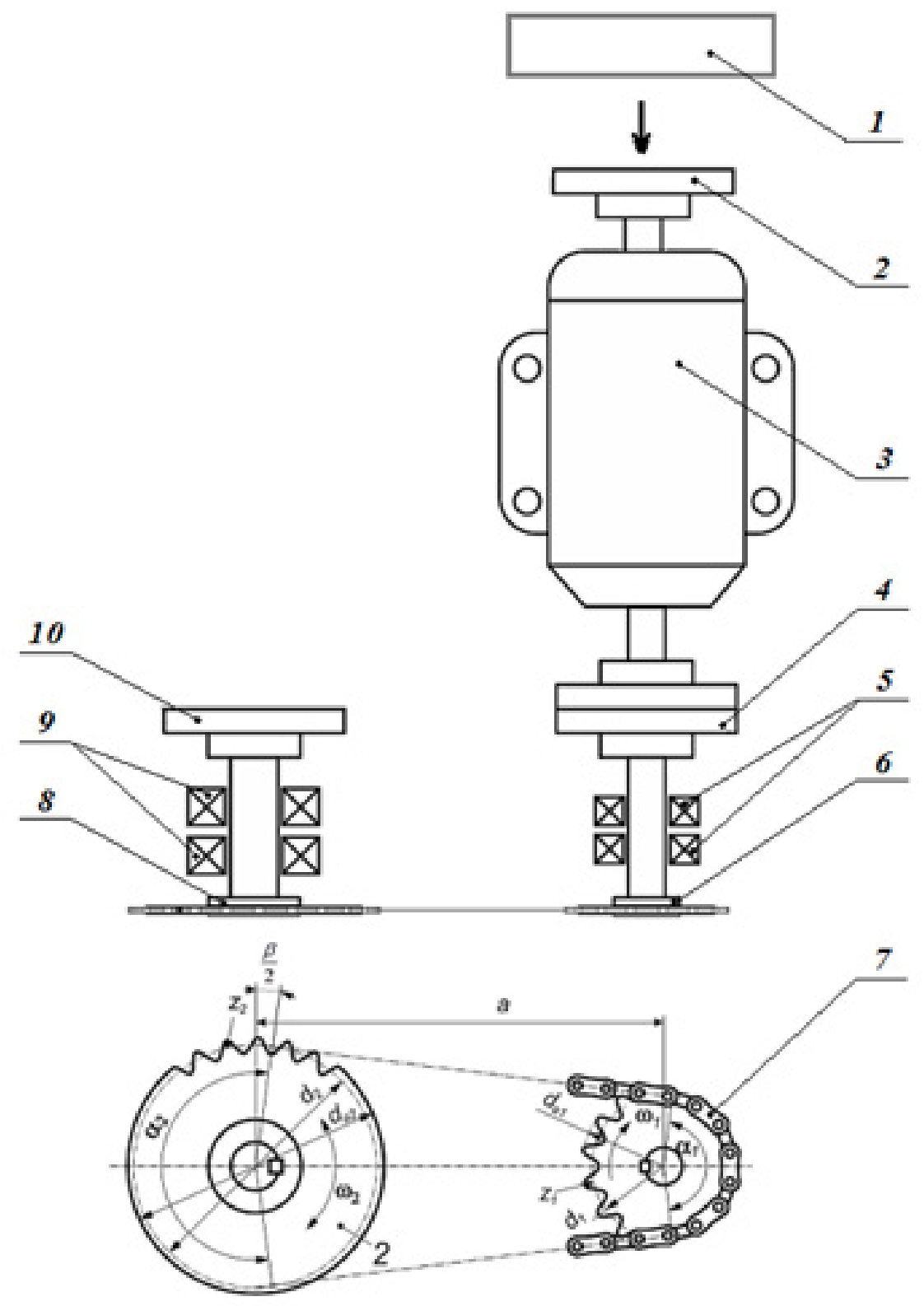

Figure 1: Scheme of an electric chain drive

1 - reference body, 2 - half-coupling for mounting a reference body, 3 - induction motor, 4 - safety clutch of the induction motor, 5 - driving shaft support bearings, 6 - driving shaft with a driving chain-wheel, 7 - chain, 8 - driven shaft with a driven chain-wheel, 9 -driven shaft support bearings, 10 - driven shaft half-coupling

I) The moment of inertia of the rotating masses of an electric chain drive taking into account losses in the chain mesh.

First, we determine the moment of inertia of the rotating masses 2-3-4-5-6-7-8-9-10 (see Fig. 1) taking into account losses in the chain mesh.

For this purpose, the moment of inertia of the electric chain drive is defined taking into account losses. At first, the angular acceleration of the driving shaft during the electric chain drive speeding up $\varepsilon 1$ is measured. Further, the reference body 1 is attached to the half-coupling 2 with fasteners. The induction motor 3 starts and the angular acceleration of the system of rotating masses with the reference body during the electric chain drive speeding up $\varepsilon^{2}$ is measured.

The angular acceleration of the motor is measured by means of an incremental encoder. The torque $\mathrm{M}$ during the first and the second starts is defined as follows.

Without the reference body 1 :

$M=\left(k_{\text {loss }} \cdot J_{C h D}\right) \cdot \varepsilon_{1}$ 
With the reference body 1 :

$$
M=\left(k_{\text {loss }} \cdot J_{\text {ChD }}+J_{\text {ref }}\right) \cdot \varepsilon_{2}
$$

Where,

$k_{\text {loss }}$ is the coefficient characterizing mechanical and added losses; $J_{C h D}$ is the moment of inertia of the electric chain drive, $\left[\mathrm{kg} \cdot \mathrm{m}^{2}\right] ; J_{\text {ref }}$ is the moment of inertia of the reference body, $\left[\mathrm{kg} \cdot \mathrm{m}^{2}\right] ; \varepsilon_{1}$ is the angular acceleration of the driving shaft without a reference body, [rad/s]; $\varepsilon_{2}$ is the angular acceleration of the driving shaft with a reference body, [rad/s];

Since losses in the stator and the rotor, the input voltage, the power frequency, and the stator resistance is not changed at the first and the second starts, therefore, the speed-torque curve of the induction motor is invariable. Hence, the right-hand parts of Eq. 1 and Eq. 2 are equal, and it is possible to find the moment of inertia of the electric chain drive taking into account mechanical and added losses:

$$
k_{\text {loss }} \cdot J_{\text {ChD }}=J_{\text {ref }} \cdot \frac{\varepsilon_{2}}{\varepsilon_{1}-\varepsilon_{2}}
$$

To determine an average value of the moment of inertia of the electric chain drive taking into account mechanical and added losses in the speed range from zero to the rated value of the angular velocity, the Eq. 3 takes the form:

$$
k_{\text {loss }} \cdot J_{C h D}=J_{\text {ref }} \cdot \frac{\overline{\varepsilon_{2}}}{\overline{\varepsilon_{1}}-\overline{\varepsilon_{2}}}=J_{\text {ref }} \cdot \frac{t_{1}}{t_{2}-t_{1}}
$$

Where,

$t_{1}$ is the time needed to speed the electric chain drive up from zero to the rated angular velocity during the first start (without the reference body), [s]; $t_{2}$ is the time needed to speed the electric chain drive up from zero to the rated angular velocity during the second start (with the reference body), [s].

Thus, knowing the values of the moments of inertia of the reference body, one can determine the moment of inertia of the electric chain drive taking into account losses by measuring only one parameter, which is the acceleration time.

From Eq. 4 it is observed that having determined the moment of inertia of the electric chain drive rotating parts without taking into account losses in the chain mesh, $J_{C h D}$, one can determine its efficiency:

$$
\eta_{\text {ChD }}=\frac{1}{k_{\text {loss }}}=\frac{J_{C h D}}{J_{\text {ref }} \cdot \frac{t_{1}}{t_{2}-t_{1}}}
$$

In Eq. $5\left(J_{\text {ref }} \cdot t_{1}\right) /\left(t_{2}-t_{1}\right)$ is greater than $J_{C h D}$ since there are always losses in the chain mesh. That is, $\left(J_{\text {ref }} \cdot t_{1}\right) /\left(t_{2}-t_{1}\right)$ is greater than the value of $J_{C h D}$ due to losses in the chain mesh, which can be expressed through the moment of inertia of chain mesh losses. Thus, the chain drive efficiency is always less than one in the presence of these losses.

II) The moment of inertia of the rotating masses of an electric chain drive without losses in the chain mesh.

In order to determine the moment of inertia of the rotating masses of the electric chain without losses in the chain mesh, it is necessary to find the moment of inertia of the chain 7 .

To determine the moment of inertia of a chain, it is needed to determine the moments of inertia of those chain parts, which are wrapped around the driving and driven chain-wheels, about the rotation axes of the respective chain-wheels. To do it, the values of the angles $\beta, \alpha_{1}$ and $\alpha_{2}$ (see Fig. 1) are determined.

The driving chain-wheel wrap perimeter is defined as follows:

$l_{\mathrm{ch} 1}=\alpha_{1} \frac{d_{1}}{2}$

Where,

$d_{1}$ is the diameter of the pitch circle of the driving chain-wheel.The driven chain-wheel wrap perimeter is defined as follows:

$$
l_{\mathrm{ch} 2}=\alpha_{2} \frac{d_{2}}{2}
$$

Where,

$d_{2}$ is the diameter of the pitch circle of the driven chain-wheel. Knowing the specific weight of a length unit of a chain mch and the pitch circle radius, one can determine the value of the moment of inertia of the chain parts wrapped around the driving and driven chain-wheels.

The moment of inertia of the chain part wrapped around the driving chain-wheel about its rotation axis is defined as follows:

$J_{\mathrm{ch} 1}=m_{\mathrm{ch}} l_{\mathrm{ch} 1}\left(\frac{d_{1}}{2}\right)^{2}$

The moment of inertia of the chain part wrapped around the driven chain-wheel about its rotation axis is defined as follows:

$$
J_{\mathrm{ch} 2}=m_{\mathrm{ch}} l_{\mathrm{ch} 2}\left(\frac{d_{2}}{2}\right)^{2}
$$


Further, the moment of inertia of the system of rotating masses 2-3-4-5-6 (see Fig. 1), $J_{1}$, is determined taking into account losses (similarly with the methodology mentioned above):

$$
k_{\text {loss } 1} \cdot J_{1}=J_{\text {ref }} \cdot \frac{\varepsilon_{4}}{\varepsilon_{3}-\varepsilon_{4}}
$$

Similarly, the moment of inertia taking into account losses of the rotating masses 2-3 (see Fig. $1), k_{\text {loss } 2} \cdot J_{i m}$, is defined. Then, having connected the induction motor to the driven shaft, the moment of inertia of the rotating masses 2-3-8-9$10, k_{\text {loss } 3} \cdot J_{i m 2}$, is defined. With the known $k_{\text {loss } 2} \cdot J_{\text {im }}$ and $k_{\text {loss } 3} \cdot J_{\text {im2 }}$, one can determine the moment of inertia of the rotating masses 8-9-10:

$$
k_{\text {loss } 4} \cdot J_{2}=k_{\text {loss } 3} \cdot J_{\text {im } 2}-k_{\text {loss } 2} \cdot J_{\text {im }}
$$

Based on the obtained data, the moment of inertia of the electric chain drive rotating parts, $J_{C H D}$, is defined according to the conventional methodology based on the law of conservation of energy.
In this case, the moment of inertia with respect to the driving shaft rotation axis is defined without regard to chain mesh losses.

$J_{\mathrm{ChD}} \cdot \frac{\omega^{2}}{2}=\left(k_{\text {loss } 1} \cdot J_{1}+J_{\mathrm{ch} 1}\right) \cdot \frac{\omega^{2}}{2}+\left(k_{\text {loss } 4} \cdot J_{2}+J_{\mathrm{ch} 2}\right) \cdot \frac{\omega^{2}}{2 i^{2}}+E_{\mathrm{k} 1}+E_{\mathrm{k} 2}$

Where,

$E_{\mathrm{k} 1}=E_{\mathrm{k} 2}=\frac{L_{\mathrm{ch}}-l_{\mathrm{ch} 1}-l_{\mathrm{ch} 2}}{2} \cdot m_{\mathrm{ch}} \cdot \frac{\left(\omega \frac{d_{1}}{2}\right)^{2}}{2}$

is the kinetic energy of driving and driven chain parts, respectively.

$$
\begin{aligned}
& J_{\mathrm{ChD}}=k_{\text {loss } 1} \cdot J_{1}+J_{\mathrm{ch} 1}+\frac{k_{\text {loss } 4} \cdot J_{2}+J_{\mathrm{ch} 2}}{i^{2}}+ \\
& +\frac{k_{\text {loss } 4} \cdot J_{2}+J_{\mathrm{ch} 2}}{i^{2}}
\end{aligned}
$$

Where,

$i$ is the gear ratio of a chain transmission, $L_{c h}$ is the total length of a chain.

III)Determining the efficiency of a chain drive. In view of Eq. 12 the Eq. 5 takes the final form:

$$
\eta_{C h D}=\frac{k_{\text {loss } 1} \cdot J_{1}+J_{\mathrm{ch} 1}+\frac{k_{\text {loss } 4} \cdot J_{2}+J_{\mathrm{ch} 2}}{i^{2}}+\left(L_{\mathrm{ch}}-l_{\mathrm{ch} 1}-l_{\mathrm{ch} 2}\right) \cdot m_{\mathrm{ch}} \cdot \frac{d_{1}^{2}}{4}}{J_{r e f} \cdot \frac{t_{1}}{t_{2}-t_{1}}}
$$

\section{THE INSTRUMENTS FOR THE CHAIN DRIVE EFFICIENCY EVALUATION}

In order to evaluate the efficiency of the chain drive with the developed method, a hardware- software system was proposed to use, which consists of an encoder, a measuring converter (registration unit) and a personal computer (PC) with a software installed, which records and analyses the digital signal (Fig. 2).

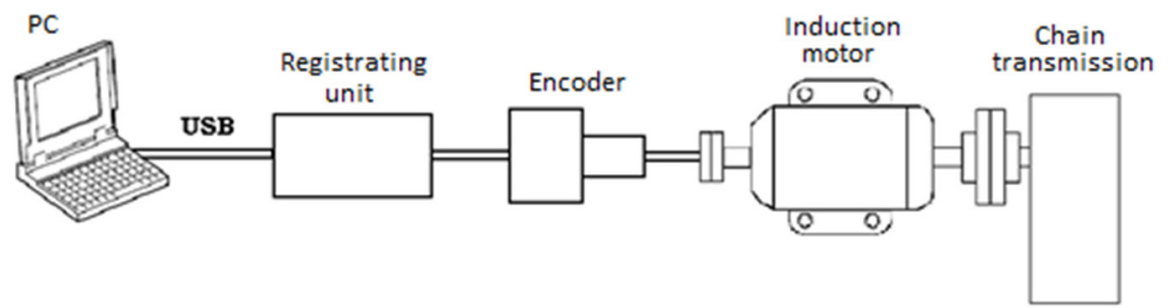

Figure 2: Structure of a hardware-software system for evaluation of the chain drive efficiency

The encoder is connected to the motor shaft by means of an adapter coupling. The operation principle of the system is based on the measurement of duration of pulses generated by the electromagnetic transducer. The electric signal is generated by the transducer when the magnetic field within its operating area changes. The change of the magnetic field is initiated by consecutive passage of the measuring disk teeth through the transducer operating area. By rotating the measuring disk, the transducer signal is generated in the form of sinusoidal pulses whose duration is proportional to the duration of passage of the transducer's area by a measurement disk tooth. The measured pulse duration is a characteristic of the motor rotation speed. The difference between consecutive measurements of the duration of two pulses is the value of the angular 
acceleration of the motor rotation. The encoder generates 5,000 pulses per a shaft revolution, which increases the measurement accuracy of the system. The fractional error of the angular acceleration measurement is $\pm 0.05 \%$. Next, the analog signal from the encoder is transferred to the registration unit input terminal. The measuring microcontroller of the registration unit fulfills measurement of the analog signal duration, and the digital signal is transferred to the USB-interface unit. Then, the array of data is transferred to the $\mathrm{PC}$ and recorded using a terminal program. The PC terminal program provides mathematical treatment of the data array and calculation of angular velocities and accelerations of the rotating motor for each tooth of the measuring disk. Based on the data of the angular accelerations obtained during the motor acceleration, both with a reference disk and without it, the moment of inertia is computed. Figure 3 shows a test bench for evaluation of the mechanical efficiency of an electric chain drive. The test bench was designed and built for testing of the developed method and its experimental substantiation.



Figure 3: The test bench for control the mechanical efficiency of a chain transmission

As a test chain a double-strand sleeve-type chain was selected with the pitch of $9.525 \mathrm{~mm}$ (2PV-9,525-17), which is used in the valve gears (VAZ 2101, 21011, 21013, 2103, 21061, 2107), the engines (GAS 3110, 3105,3102 ) and other mechanisms. The number of teeth of the drive chain-wheel was $z=23$, the number of teeth of the driven chain-wheel was $z=38$. Preload chain tension was adjusted by moving a support of the driven shaft along the tracks parallel to the chain-wheels plane. In experiments we used a voltage stabilizer Saturn SNE-O-10 (11 kVA, 50 A) with a relative error of stabilizing of $0.5 \%$.

\section{RESULTS \\ Experimental Validation of Data Obtained with the Proposed Method}

For the experimental validation of data obtained with the proposed method we compared the values of the torque obtained with the developed method and with the torque sensor M40-100 during the induction motor acceleration. The experiments were carried out according to the scheme shown in Fig. 4. Since the torque sensor 3 measures the torque, MTS, at the point where it is mounted, we used the following equation in order to determine the torque value, which measures a sensor, by the developed method:

$$
M_{D M}=M-J_{\text {im }} \cdot \varepsilon
$$

Where,

$M$ is the torque of the induction motor, $[\mathrm{N} \cdot \mathrm{m}] ; \mathrm{J}_{\text {im }}$ is the moment of inertia of the induction motor, $\left[\mathrm{kg} \cdot \mathrm{m}^{2}\right] ; \varepsilon$ is the angular acceleration of the driving shaft, $\left[\mathrm{rad} / \mathrm{s}^{2}\right]$.


Figure 4: Scheme to measure the electric chain drive torque with a torque sensor M40-100

1 - motor shaft; 2 - safety coupling; 3 - torque sensor M40-100; 4 - PC with installed software;

5 - driven shaft with a coupling; 6 - loading rotary body

In the experimental part of this work in the application of both methods 10 measurements were carried out, blunders were eliminated, random errors were determined and the average torque values were found. Table 1 shows the values of random errors of the torque measurement in the application of torque sensors (TS) and the developed (DM) method. Table 2 shows the values of the average torque obtained. According to Table 2 , we can conclude that the torque values obtained by the developed method and using the torque sensor differ from each other by a maxi- 
mum relative error, $\delta$, equal to $1.2 \%$, indicating a reproducibility of the measurements obtained by different methods. However, it should be noted that the accuracy of the torque measurement by the developed method is somewhat higher than that when using a torque sensor (see Table 1).
This may be due to the time required to recover elastically deformed state of the strain gauge body. In turn, the frequency of signal registration (response to changing conditions) with the use of the developed method is much higher than the strain gauge speed.

Table 1: Values of the random errors of the torque measurement in the application of torque sensors (TS) and the developed (DM) method

\begin{tabular}{|c||c|c|c|c|c|c|c|}
\hline \multirow{2}{*}{ Variable } & \multicolumn{7}{|c|}{ Number of revolutions, [rev/min] } \\
\cline { 2 - 8 } & $250-400$ & $400-500$ & $500-600$ & $600-700$ & $700-800$ & $800-850$ & $850-950$ \\
\hline$\delta$ (MDM), [\%] & 0.4 & 0.4 & 0.5 & 0.4 & 0.5 & 0.5 & 0.5 \\
\hline$\delta$ (MTS), [\%] & 0.7 & 0.9 & 0.8 & 0.6 & 0.8 & 0.8 & 0.9 \\
\hline
\end{tabular}

Table 2: The comparison of the average torque values within selected speed range obtained with the developed method (DM) and using torque sensors (TS)

\begin{tabular}{|c|c|c|c|c|c|c|c||}
\hline \multirow{2}{*}{ Variable } & \multicolumn{7}{|c|}{ Number of revolutions, [rev/min] } \\
\cline { 2 - 8 } & $250-400$ & $400-500$ & $500-600$ & $600-700$ & $700-800$ & $800-850$ & $850-950$ \\
\hline $\mathrm{M}_{\mathrm{DM}},[\mathrm{Nm}]$ & 5.262 & 7.438 & 9.420 & 11.555 & 13.886 & 14.515 & 13.582 \\
\hline $\mathrm{M}_{\mathrm{TS}},[\mathrm{Nm}]$ & 5.320 & 7.356 & 9.498 & 11.620 & 14.034 & 14.686 & 13.692 \\
\hline$\delta,[\%]$ & 1.1 & -1.1 & 0.8 & 0.6 & 1.1 & 1.2 & 0.8 \\
\hline
\end{tabular}

A random error in the application of the proposed method is likely related to the instability of the input voltage.

\section{CONCLUSIONS}

A significant advantage of the proposed method is the possibility to evaluate the efficiency of a chain drive with a high measuring frequency rate within a wide range of possible modes of operation as it uses the angular acceleration and the acceleration time as controlled variables. The proposed method involves the use of loading rotary bodies attached to a driven shaft of a chain drive to create the rated force in a chain, eliminating a need for a loader. The developed method solves the problem of determining the mechanical efficiency of chain transmissions without taking into account losses in their bearing units. The developed method can be used by companies that manufacture chain drives. Determining acceptable levels of the acceleration time of chain drives, it is possible to evaluate their mechanical efficiency, which is influenced by a build quality, lubrication and materials used. It is recognizing that the mechanical efficiency of chain transmissions characterizes degradation processes occurring in their elements, and therefore may be a criterion of their lifetime. This method can also be applied in studies of chain transmissions, which target detection of factors influencing their energy performance.

\section{REFERENCES}

1) Conwell, J. C., Johnson, G. E. (1996). Experimental investigation of link tension and roller sprocket impact forces in roller electric chain drives. Mechanism and Machine Theory, 31 (4), 533-544.

2) Davies, D. N. C., Gustafsson, K. G., Owen, P. J., Nordkvist, K. E. (1981). Roller Chain as a Transfer Drive for the Automobile. J. Mech. Design 103(1), 19-28.

3) Liu, S.P., Wang, K. W., Hayek, S. I. (1990). Modeling and analysis of electric chain drive systems. J. Acoust. Soc. Am. Vol. 87, Issue S1, 136-139.

4) Spicer, J. B., Richardson, C. J. K., Ehrlich, M. J., Bernstein, J. R., Fukuda, M., Terada, M. (1999). Effects of Frictional Loss on Bicycle Electric chain drive Efficiency. J. Mech. Design. 123(4), 598-605.

5) Spicer, J. B., Richardson, C. J. K., Ehrlich, M. J., Bernstein, J. R., Fukuda, M., Terada, M. (2000). On the efficiency of bicycle electric chain drives. Tech. J. of the IHPVA, 50 (2000), 3-9.

6) Troedsson, I., Vedmar, L. (1999). A Method to Determine the Static Load Distribution in an electric chain drive. ASME J. Mech. Design, 121(3), 402-408.

7) Vorobyev, N.V. (1968) Tsepnye peredatshi, Moscow: Mashinostroeniye.

8) Zhang, L., Zhang, C., Horng, J.-H., Chen, Z. (2012). Study on Simulation of the Chain Transmission Mechanism. Advanced Materials Research, 593 (6), 797-800.

Paper sent to revision: 21.09.2015.

Paper ready for publication: 14.12.2015.

Journal of Applied Engineering Science 13(2015)4, 341 